\title{
Pathway redesign: putting patients ahead of professionals
}

\author{
Author: Michael PW Grocott ${ }^{\mathrm{A}}$
}

Many perioperative clinical pathways, and therefore patient journeys, are focused around provider, rather than patient, convenience. Business process re-engineering (BPRE) offers a framework for transformative process-change with the aim of improving 'consumer experience' and efficiency and may be an effective driver for improving patient experience and value within healthcare. Involvement of patients in service and pathway design, through experience-based codesign, is increasingly prevalent and may be an effective complement to BPRE. The elective perioperative pathway offers an opportunity to rethink the patient journey with the aim of maximising opportunities for effective shared decision making and improving preparation for surgery through prehabilitation and management of longterm conditions (comorbidity/multimorbidity management). Additional opportunities include improved management of transitions of care and effective medicines management to minimise polypharmacy. Pathway mapping, deconstruction and reconstruction enables such changes and is a method of service transformation that may have relevance for a spectrum of other elective/scheduled pathways.

KEYWORDS: Perioperative, pathway redesign, shared decision making, prehabilitation, comorbidity

\section{Introduction}

It is an uncomfortable truth for those of us who believe that we practice patient-centred care that, in many cases, clinical pathways, and therefore patient journeys, have a structure that is related more to the convenience of the care providers than that of the patients. The timing, location and manner of interactions between patients and healthcare providers are frequently dictated by provider priorities, including convenience, habit and economies of scale, rather than patient wishes. Control of the pathway resides almost exclusively with the providers, not those being provided for.

Patients may, from choice, prefer to have elements of healthcare at a time or location that is more convenient to them. They may want to have some control of how decisions are made and communicated and they may wish to utilise technology to facilitate some of their healthcare needs, rather than rely exclusively on face-to-

Author: ${ }^{\text {p }}$ rofessor of anaesthesia and critical care medicine, University of Southampton, Southampton, UK and Acute, Critical and Perioperative Care Research Group, Southampton, UK face interactions. Innovations to support such interactions are spreading rapidly within some areas of medicine through the use of telemedicine, m-health and 'wearables'. ${ }^{1-3}$ However, arguably, hospital-based procedural specialties, such as surgery, are progressing more slowly than others.

The notion of silos within healthcare has become a cliché, but the issue that is reflected in this cliché, that care providers tend to think and act in relation to their immediate physical and organisational surroundings, is of particular relevance when considering elective/scheduled care pathways. Health financing mechanisms may reinforce 'silo behaviour'. Fee for service mechanisms, where funding is allocated based on the number and type of activities delivered (probably still the most common funding mechanism in US healthcare), tend to incentivise activity and focus personal and institutional efforts on supporting this. ${ }^{4}$ Capitation mechanisms, on the other hand, where funding is allocated on a per capita basis to support the healthcare needs of a population, tend to incentivise value and thereby focus efforts on cost containment and efficiency across the whole patient pathway. $^{4}$

This article will briefly summarise the challenges of perioperative care, then focus on patient perspectives on clinical pathways / patient journeys as well as patient codesign, and finally explore the notion of pathway redesign. Within this framework, opportunities to facilitate shared decision making, prehabilitation and comorbidity management will be discussed.

Surgery for solid tumours offers an excellent example around which this discussion will be framed, but the issues are of relevance to a variety of other healthcare contexts including other cancer treatments (systemic anticancer treatments and radiotherapy).

\section{The challenge of perioperative care}

The global burden of surgery is estimated to exceed 300 million operations per year and national surgical volume is associated with the relative wealth of a nation; in the UK we undertake around 4 million surgeries annually. ${ }^{5,6}$ In the global context, death within 30 days of surgery is a substantial public health challenge presenting a burden of attributable mortality greater than that attributed to malaria, HIV and tuberculosis combined. ${ }^{7}$ Moreover, for every patients that dies, there are approximately 10-fold more who will experience substantial postoperative morbidity and the consequences of that morbidity are threefold: patient suffering, excess long-term mortality and inefficient use of limited resources. ${ }^{8-12}$ Addressing this burden through improving perioperative care is an important priority globally. 
Process re-engineering: mapping, deconstruction and reconstruction

Business process re-engineering is a business management approach focused on the analysis and redesign of workflows and processes with the aim of improving 'customer experience' and improving efficiency and thereby lowering costs. ${ }^{13,14}$ These aims clearly resonate in the resource-constrained environment of healthcare delivery. ${ }^{15,16}$ While the language of "customer experience' may sit uncomfortably with healthcare providers, the importance of prioritising patients' needs, outcomes and experience within healthcare is seen as self-evident. The concepts of personalised care and patients' control over their own health are central to The NHS Long Term Plan and initiatives including personal health budgets, 'Choosing Wisely' and NHS Choices are symptomatic of a clear transition from paternalistic to patientcentred healthcare. ${ }^{17}$ The importance of maximising value through improving service efficiency is also central to The NHS Long Term Plan and fundamental to sustainable healthcare in an environment of limited healthcare resources facing relentlessly increasing demand. The ineffectiveness of incremental cost saving and service refinement to meet the rapidly evolving face of healthcare has led to the drive for transformational change - pathway redesign addresses this challenge. In practice, re-engineering involves processes of mapping, deconstruction and reconstruction in order to define a more patient-focused and efficient (with respect to value) process. In common with other pathway-focused initiatives (eg enhanced recovery), undertaking these processes within the context of a multidisciplinary group can be highly effective in terms of promoting engagement with the re-engineered pathway. ${ }^{18}$

\section{Patient perspectives and codesign}

From a patient perspective, expectations of what a perioperative pathway might offer are relatively uncomplicated. Consistent with the focus on patient-centred care, most patients expect to be a partner in the decisions made about them and the delivery of care. Taking this starting point, four key questions pertain to the surgical journey: has the right decision been made, am I as well prepared as possible, will I be properly (and safely) cared for and will I fully recover? ${ }^{15}$ From a provider perspective, these questions map neatly onto shared decision making, prehabilitation and comorbidity management, safe and effective hospital care, and rehabilitation, respectively.

The idea of codesign between professionals and patients, also known as experience-based codesign (EBCD), is increasingly prevalent in healthcare transformation. ${ }^{19}$ The value of the 'lived experience' of patients and public in informing the design of patient pathways has been demonstrated in a variety of contexts across the healthcare spectrum, including primary and secondary care and integrated pathways linking primary and secondary care. $^{20-22}$ Clinical conditions have encompassed malignant disease, non-malignant disease, mental health problems, and palliative and end-of-life care, and EBCD is likely to be of value in many more conditions and contexts. ${ }^{19-24}$ Examples of codesign innovations include increased patient self-management with reduced clinic appointments, better patient information resources, buddy systems, flexible clinic appointment scheduling and improved transfers of care. ${ }^{22,25,26}$
Effective codesign often evolves into ongoing partnership and patients may be more likely to engage with (and adhere to) programmes that were codesigned and are therefore inherently aligned with their needs.

\section{Pathway redesign before surgery}

There are three key determinants of outcome following surgery: the patient, their pathology and the type of surgery undertaken. The index pathology requiring surgery may be difficult to modify prior to the procedure, although increasingly neoadjuvant therapy may be important prior to cancer surgery. On the other hand, given sufficient time, there are opportunities to improve patient health through prehabilitation and/or comorbidity management and to inform choices about surgery through effective shared decision making (SDM). ${ }^{27}$ However, as currently configured, preoperative pathways do not typically enable these opportunities; the pathway is typically focused on diagnosis and characterisation of the primary pathology in order to define the treatment required. Consequently, opportunities are missed to characterise and improve patient health and to facilitate more effective decision-making. Enabling these activities involves parallel work-streams focusing on patient and pathology that are brought together in the shared decision-making process, around the time of multidisciplinary team (MDT) meeting, as illustrated in the contrasting pathways presented in Figs $1 a$ and $b .^{16}$

Essential to achieving this goal is early engagement between care providers and patients that is focused on 'patient staging' (in contrast to 'pathology staging') in order to maximise the window of opportunity for informed decision making and intervention before surgery. For fit healthy patients without significant comorbidities, this interaction may be a simple screening process followed by signposting down a low-risk route, with health advice and expectations around surgery delivered in a 'surgery school' environment. For patients who may have a higher risk of adverse outcome, screening may indicate the requirement for further assessment and needs-based prescription of relevant interventions. A variety of assessment tools are available and may be configured around a 'high-risk' or 'shared decision-making' clinic. ${ }^{28}$ This clinic enables integration of information to guide prehabilitation and comorbidity management. In some cases, it also provides the forum for important discussions about the harms and benefits of surgery through SDM.

Other preoperative opportunities to improve patient pathways relate to better management of transitions of care, particularly between primary/community and secondary care, and effective medicines management. Both of these areas are, arguably, equally of relevance following surgery and will be discussed in that context below.

\section{Shared decision making}

The landmark Royal College of Anaesthetists' publication Perioperative medicine: The pathway to better surgical care ${ }^{29}$ defined the perioperative epoch as 'from the moment of contemplation of surgery until full recovery'. Crucially, this formulation encompasses the notion of contemplation of surgery, and therefore implicitly acknowledges that not having surgery may be in the patients' best interests. Shared (or collaborative) decision making provides the process through which the relative benefits and harms of surgery (and other interventions) can be defined, communicated and considered in order to arrive at the best decision for a particular patient at a 
a

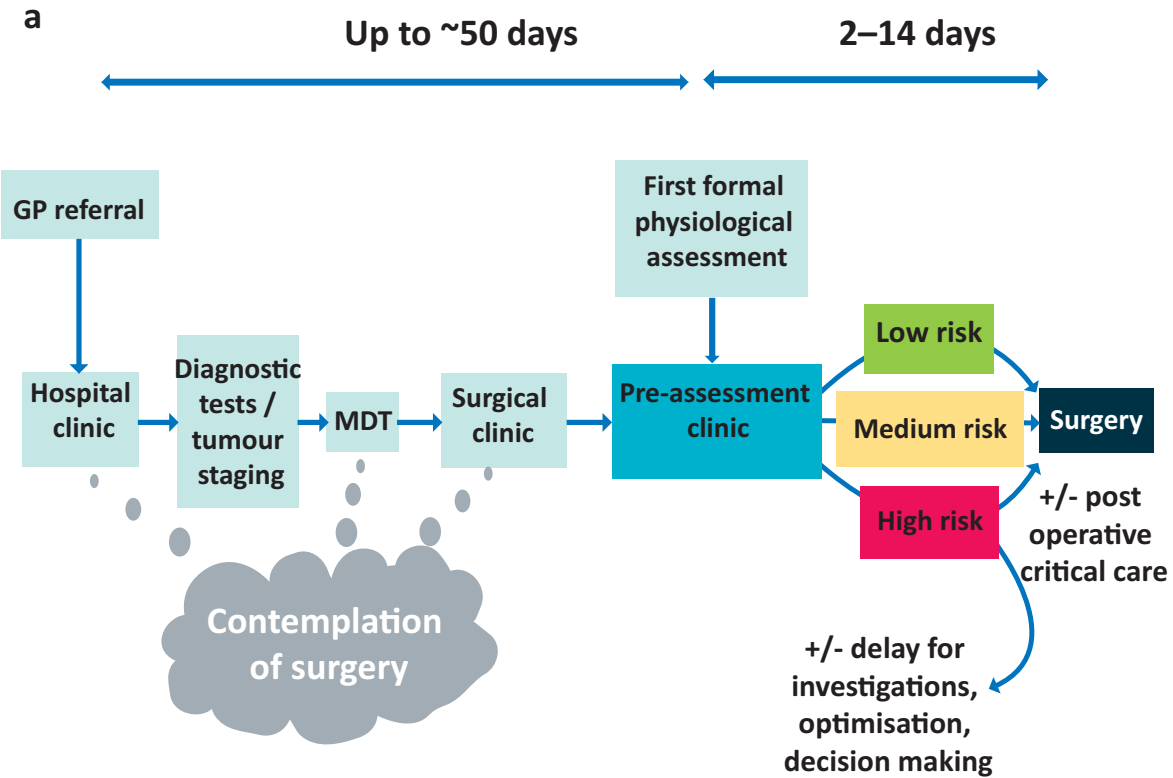

b

Up to 62 days

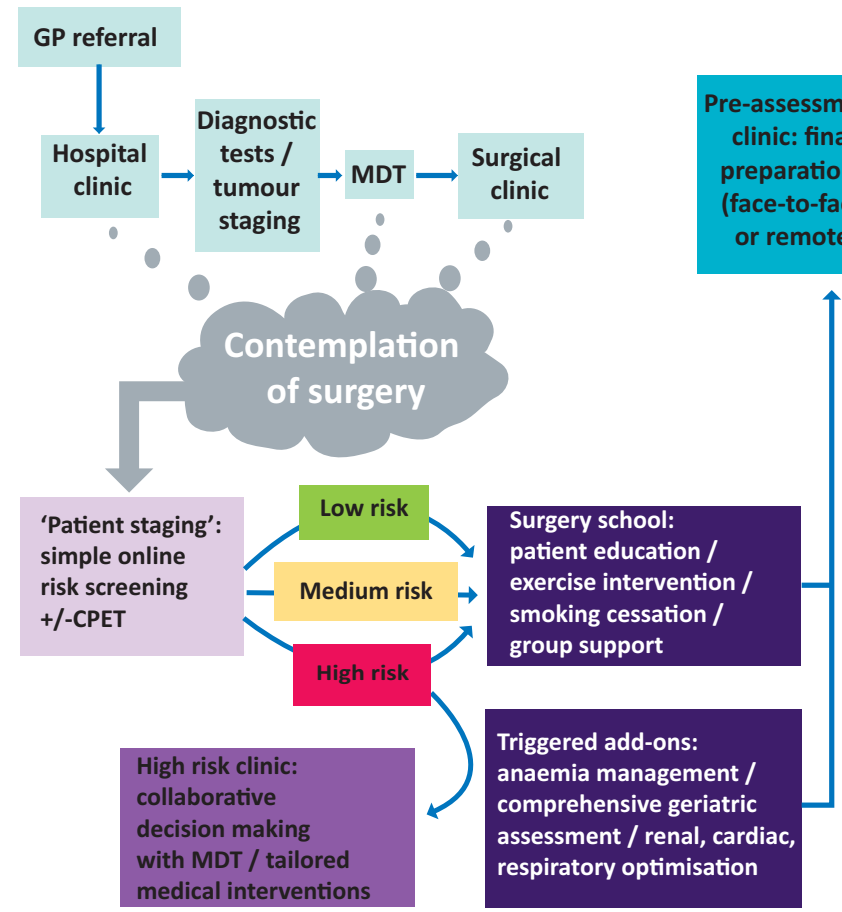

Fig 1. Contrasting perioperative pathways. a) Traditional model of the journey from general practitioner referral through to surgery. b) The pathway 're-engineered'an example of process deconstructionreconstruction in perioperative pathways. Adapted with permission from Grocott MPW, Plumb JOM, Edwards M, Fecher-Jones I, Levett DZH. Re-designing the pathway to surgery: better care and added value. Perioper Med (Lond) 2017;6:9 and under the terms of the Creative Commons Attribution 4.0 International License. $\mathrm{CPET}=$ cardiopulmonary exercise testing; $\mathrm{GP}=$ general practitioner; $\mathrm{MDT}=$ multidisciplinary team. particular time. ${ }^{30}$ Context is all important: what might be a trivial decision for a young fit individual may seem very different following a debilitating long-term illness when likelihood of harm may be substantially increased and the benefits from a good outcome may be diminished. Critically, effective SDM is dependent on the availability of relevant information and the opportunity for reflection. Strikingly, the traditional anaesthetic preoperative assessment clinic occurring shortly before the planned procedure does not serve this purpose. By this time the patient will have had clear expectations of surgery set for a substantial period of time and the opportunity for SDM may have been lost. On the other hand, early identification and evaluation of individuals that may be at particular risk from harm around the time of surgery occurring in parallel to the "work up' for the surgical pathology can effectively support SDM so that information on 'patient staging' and 'pathology staging' can be brought together before any definitive decisions have been made and the patient involved in a balanced discussion of likely benefit and harm framed around their own life context and appetite (or 
not) for risk. SDM is clearly of benefit to patients, in that they will not inadvertently head down a path that they would not wish to when fully informed. Importantly, SDM is an opportunity not a mandate. Some patients prefer the paternalistic model of medical decision making and naturally defer to clinicians' advice, but even in these cases, a re-engineered pathway offers an opportunity for this to take place with all necessary information available. On the other hand, some patients wish to be centrally involved in the decision-making process, and when fully informed may on occasions make rational decisions that differ from those that their clinician team might have chosen for them.

\section{Prehabilitation}

Proactively improving functional capacity, or physiological resilience, prior to a planned physiological challenge such as major surgery is known as prehabilitation. ${ }^{31}$ We now recognise that this is part of a continuum of rehabilitation practice spanning preventative, restorative, supportive and palliative intervention, and corresponds to the diagnosis, treatment, recovery and long-term phases of many chronic conditions. The principle areas of focus of prehabilitation are activity and exercise, nutrition, psychological wellbeing, and smoking and alcohol cessation. ${ }^{31}$ While changes in behaviour are often challenging in day-today life, the presurgical epoch represents a special (arguably unique) opportunity - a 'teachable moment' - when patients are particularly focused on their own health. ${ }^{31}$

The prehabilitation literature has principally grown up around perioperative care but is increasingly seen to be applicable to any elective/scheduled care pathways. Recently, attention has been particularly focused on nonsurgical cancer treatments including systemic anticancer treatments (chemotherapy and immunotherapy) and radiotherapy. ${ }^{32}$ Prehabilitation is seen to offer patients three benefits: personal empowerment, physiological resilience and longer-term health. ${ }^{32}$ Patients comment most about the sense of personal empowerment, at a time when they often feel powerless to control their own destiny, and this has been shown to result in improved quality of life. ${ }^{33}$ Improved physiological resilience facilitates more rapid and effective recovery following treatment and may enable access to treatments that were previously inaccessible due to relative limitation of physical status. ${ }^{34}$ Finally, the enticing long-term promise of prehabilitation is the possibility of sustained behavioural change and consequent improvements in long-term health.

\section{Optimising long-term conditions}

Long-term conditions are increasingly prevalent as life-expectancy increases and more than half the population have at least one comorbidity by the age of 50 years and at 65 years, the majority are multimorbid. ${ }^{35}$ Comorbidity and multimorbidity increase the risk associated with major surgery including the likelihood of morbidity and mortality. Management of these long-term conditions is an important element of patient preparation before surgery, but faces the same challenges as prehabilitation. Where attention is only focused on comorbidity management shortly before surgery, as is typically the case with anaesthetic preassessment clinics, the limited subsequent time available before surgery is rarely sufficient for impactful intervention. The alternative approach outlined above, whereby all patients are screened as soon as possible after the 'moment of contemplation of surgery', offers the opportunity to intervene in good time and avoid either delaying surgery or failing to optimise management. In this context, dedicated perioperative clinics are becoming increasingly prevalent. Perioperative anaemia clinics are probably the most common, they may be virtual or face-to-face, and typically function around a management algorithm agreed between relevant specialist and applied by members of the perioperative team. ${ }^{36}$ For the example of anaemia, haemoglobin levels are checked well before surgery and abnormal values automatically trigger a haematinic screen. The majority of patients are found to have iron deficiency or functional anaemia and may be managed by the perioperative care team through prescription of oral or intravenous iron, with sufficient time often being available for substantial improvements in haemoglobin levels. More complex cases may be referred directly to the face-to-face haematology clinic for rapid follow-up. The business case for such anaemia clinics is often very straightforward due to the cost savings associated with reduced blood transfusion. ${ }^{36}$ While other business cases may be more challenging there are a variety of such clinics now appearing including perioperative diabetes, heart failure, chronic obstructive pulmonary disease and implantable device clinics. ${ }^{15}$

\section{After surgery}

The postoperative pathway also offers a number of other opportunities for service redesign including rehabilitation, management of long-term conditions, medicine management and managing transitions of care. The effective implementation of postoperative enhanced recovery through the use of monitoring of simple patient focused measures, such as the 'DrEaM' (drinking, eating and mobilising) concept, is an excellent example of previous pathway redesign. ${ }^{37}$ Transitions of care rely on effective communication, which may be facilitated by patients and their carers, and examples of patient codesign to improve care transitions are available. ${ }^{38,39}$ Prescribing issues, either due to simple transcription errors at the multiple points where drugs have to be re-prescribed during the perioperative journey, or due to change in patient condition that render previous prescriptions redundant are common. Medicines management, or 'deprescribing', can effectively reduce polypharmacy, mitigate simple errors in prescription and contribute to important initiative such as opioid minimisation campaigns. ${ }^{40}$

\section{Conclusion}

Business process re-engineering offers a framework for transformative process change and may be an effective driver for improving patient experience and value within healthcare. Perioperative pathways are typically focused around provider, rather than patient convenience, and involvement of patients in service and pathway design through experience-based codesign may be of value. Opportunities for effective SDM and improving preparation for surgery through prehabilitation and management of long-term conditions (comorbidity/multimorbidity) may be facilitated by pathway redesign.

\section{References}

1 Kahn JM. Virtual visits - confronting the challenges of telemedicine. N Engl J Med 2015;372:1684-5.

2 Brørs G, Pettersen TR, Hansen TB et al. Modes of e-health delivery in secondary prevention programmes for patients with coronary artery disease: a systematic review. BMC Health Serv Res 2019;19:36. 
3 Bahadori S, Immins T, Wainwright TW. A review of wearable motion tracking systems used in rehabilitation following hip and knee replacement. J Rehabil Assist Technol Eng 2018;5:1-8.

4 Simoens S, Giuffrida A. The impact of physician payment methods on raising the efficiency of the healthcare system: an international comparison. Appl Health Econ Health Policy 2004;3:39-46.

5 Weiser TG, Haynes AB, Molina G et al. Estimate of the global volume of surgery in 2012: an assessment supporting improved health outcomes. Lancet 2015;385(Suppl 2):S11.

6 Fowler AJ, Abbott TEF, Prowle J, Pearse RM. Age of patients undergoing surgery. $\mathrm{Br}$ J Surg 2019;106:1012-8.

7 Nepogodiev D, Martin J, Biccard B et al. Global burden of postoperative death. Lancet 2019:393:401.

8 Dindo D, Demartines N, Clavien PA. Classification of surgical complications: a new proposal with evaluation in a cohort of 6,336 patients and results of a survey. Ann Surg 2004;240: 205-13.

9 Grocott MP, Browne JP, Van der Meulen ] et al. The postoperative morbidity survey was validated and used to describe morbidity after major surgery. J Clin Epidemiol 2007;60:919-28.

10 Khuri SF, Henderson WG, DePalma RG et al. Determinants of longterm survival after major surgery and the adverse effect of postoperative complications. Ann Surg 2005;242:326-41.

11 Moonesinghe SR, Harris S, Mythen MG et al. Survival after postoperative morbidity: a longitudinal observational cohort study. $\mathrm{Br}$ ] Anaesth 2014;113:977-84.

12 Eappen S, Lane BH, Rosenberg B et al. Relationship between occurrence of surgical complications and hospital finances. JAMA 2013:309:1599-606

13 Hammer M. Reengineering work: don't automate, obliterate. Harvard Business Review 1990:104-12.

14 Abrahamson E. Management fashion. Academy of Management Review 1996;21:254-85.

15 Grocott MPW, Edwards M, Mythen MG, Aronson S. Peri-operative care pathways: re-engineering care to achieve the 'triple aim'. Anaesthesia 2019;74(Suppl 1):90-9.

16 Grocott MPW, Plumb JOM, Edwards M, Fecher-Jones I, Levett DZH. Re-designing the pathway to surgery: better care and added value. Perioper Med (Lond) 2017;6:9.

17 Santhirapala R, Fleisher LA, Grocott MPW. Choosing Wisely: just because we can, does it mean we should? $\mathrm{Br}$ ] Anaesth 2019:122:306-10.

18 Roulin D, Najjar P, Demartines N. Enhanced recovery after surgery implementation: from planning to success. J Laparoendosc Adv Surg Tech A 2017:27:876-9.

19 Tsianakas V, Robert G, Maben J et al. Implementing patientcentred cancer care: using experience-based co-design to improve patient experience in breast and lung cancer services. Support Care Cancer 2012;20:2639-47.

20 Litchfield I, Bentham L, Hill A et al. The impact of status and social context on health service co-design: an example from a collaborative improvement initiative in UK primary care. BMC Med Res Methodol 2018;18:136.

21 Rawson TM, Moore LSP, Castro-Sanchez E et al. Development of a patient-centred intervention to improve knowledge and understanding of antibiotic therapy in secondary care. Antimicrob Resist Infect Control 2018;7:43.

22. Zimbudzi E, Lo C, Robinson $T$ et al. The impact of an integrated diabetes and kidney service on patients, primary and specialist health professionals in Australia: A qualitative study. PLoS One 2019;14:e0219685.
23 Matthews E, Cowman M, Denieffe S. Using experience-based co-design for the development of physical activity provision in rehabilitation and recovery mental health care. J Psychiatr Ment Health Nurs 2017;24:545-52.

24 Borgstrom E, Barclay S. Experience-based design, co-design and experience-based co-design in palliative and end-of-life care. BMJ Support Palliat Care 2019;9:60-6.

25 Khodambashi S, Haugland D, Ellingsberg A et al. An experimenta comparison of a co-design visualizing personal drug information and patient information leaflets: usability aspects. Stud Health Technol Inform 2017;245:748-52.

26 Brady GC, Goodrich J, Roe JWG. Using experience-based co-design to improve the pre-treatment care pathway for people diagnosed with head and neck cancer. Support Care Cancer 2019:[e-pub ahead of print].

27 Glance LG, Osler TM, Neuman MD. Redesigning surgical decision making for high-risk patients. N Engl J Med 2014;370:1379-81.

28 Dhesi JK, Swart M. Specialist pre-operative assessment clinics. Anaesthesia 2016;71(Suppl 1):3-8.

29 Royal College of Anaesthetists. Perioperative medicine: The pathway to better surgical care. London: RCoA, 2015.

30 Levett DZ, Edwards M, Grocott M, Mythen M. Preparing the patient for surgery to improve outcomes. Best Pract Res Clin Anaesthesiol 2016;30:145-57.

31 Richardson K, Levett DZH, Jack S, Grocott MPW. Fit for surgery? Perspectives on pre-operative exercise testing and training. Br J Anaesth 2017;119(Suppl 1):i34-i43.

32 Macmillan Cancer Support, NIHR Cancer and Nutrition Collaboration, Royal College of Anaesthetists. Prehabilitation for people with cancer: Principles and guidance for prehabilitation within the management and support of people with cancer. Macmillan, 2019. www.macmillan.org.uk/assets/prehabilitationguidance-for-people-with-cancer.pdf [Accessed 13 August 2019].

33 Brunet J, Burke S, Grocott MP, West MA, Jack S. The effects of exercise on pain, fatigue, insomnia, and health perceptions in patients with operable advanced stage rectal cancer prior to surgery: a pilot trial. BMC Cancer 2017:17:153.

34 Barberan-Garcia A, Ubré M, Roca ] et al. Personalised prehabilitation in high-risk patients undergoing elective major abdominal surgery: a randomized blinded controlled trial. Ann Surg 2018;267:50-6.

35 Barnett K, Mercer SW, Norbury M et al. Epidemiology of multimorbidity and implications for health care, research, and medical education: a cross-sectional study. Lancet 2012;380:37-43.

36 Guinn NR, Guercio JR, Hopkins T] et al. How do we develop and implement a preoperative anemia clinic designed to improve perioperative outcomes and reduce cost? Transfusion 2016;56:297-303.

37 Levy N, Mills P, Mythen M. Is the pursuit of DREAMing (drinking, eating and mobilising) the ultimate goal of anaesthesia? Anaesthesia 2016;71:1008-12.

38 Kennedy JM, van Rij AM, Spears GF, Pettigrew RA, Tucker IG. Polypharmacy in a general surgical unit and consequences of drug withdrawal. Br J Clin Pharmacol 2000;49:353-62.

39 Barnett NL, Oboh L, Smith K. Patient-centred management of polypharmacy: a process for practice. Eur J Hosp Pharm 2016;23:113-17.

40 Kelly M. Faith in subtraction: deprescribing in older patient facilitates needed surgery. Am Fam Physician 2018;98:634.

Address for correspondence: Prof Michael Grocott, University Hospital Southampton NHS Foundation Trust, Tremona Road, Southampton, Hampshire S016 6YD, UK. Email: mike.grocott@soton.ac.uk 